\title{
Finite Temperature Theory of Metastable Anharmonic Potentials
}

\author{
Marco Zoli \\ Istituto Nazionale Fisica della Materia - Dipartimento di Fisica \\ Universitá di Camerino, 62032, Italy. - marco.zoli@unicam.it
}

(Dated: November 24, 2018)

\begin{abstract}
The decay rate for a particle in a metastable cubic potential is investigated in the quantum regime by the Euclidean path integral method in semiclassical approximation. The imaginary time formalism allows one to monitor the system as a function of temperature. The family of classical paths, saddle points for the action, is derived in terms of Jacobian elliptic functions whose periodicity sets the energy-temperature correspondence. The period of the classical oscillations varies monotonically with the energy up to the sphaleron, pointing to a smooth crossover from the quantum to the activated regime. The softening of the quantum fluctuation spectrum is evaluated analytically by the theory of the functional determinants and computed at low $T$ up to the crossover. In particular, the negative eigenvalue, causing an imaginary contribution to the partition function, is studied in detail by solving the Lamè equation which governs the fluctuation spectrum. For a heavvy particle mass, the decay rate shows a remarkable temperature dependence mainly ascribable to a low lying soft mode and, approaching the crossover, it increases by a factor five over the predictions of the zero temperature theory. Just beyond the peak value, the classical Arrhenius behavior takes over. A similar trend is found studying the quartic metastable potential but the lifetime of the latter is longer by a factor ten than in a cubic potential with same parameters. Some formal analogies with noise-induced transitions in classically activated metastable systems are discussed.

PACS numbers: 03.65.Sq, 03.75.Lm, 05.30.-d, 31.15.xk
\end{abstract}




\section{Introduction}

Occuring in a broad class of natural phenomena metastability has been intensively studied in different research fields ranging from condensed matter to chemical physics, from particle and nuclear physics to cosmology. While the mathematics of the metastable phase in statistical physics was elaborated by Langer [1] forty years ago, the thorough extension of the formalism to the quantum field theory came in the seventies of the last century [2, 3, 4]. As the semiclassical approximation is known to be appropriate [5] to deal with quantum tunneling, the path integral method in the Euclidean version [6, 7] has emerged as the fundamental tool for quantitative theories of the decay rate $\Gamma$ of a metastable state. The latter is given in semiclassical theory by

$$
\Gamma=A \exp (-B / \hbar)[1+O(\hbar)]
$$

where $A$ and $B$ depend on the specific shape of the potential. In the path integral approach, $B$ is the Euclidean action calculated at the classical path around which the action varies the least while $A$ is obtained by summing over the quantum fluctuations paths. In quadratic approximation the quantum fluctuations are decoupled and their contribution can be evaluated by the theory of the functional determinants which implements the path integral method [8].

Eq. (11) is formally identical to the tunneling energy in the bistable $\phi^{4}$ potential [9] which admits an instanton [10, 11] as the classical path interpolating between the two vacua. The fundamental difference lies however in the fact that the instanton minimizes the Euclidean action whereas the classical path in the metastable potential, the bounce, is a saddle point for the action. Hence the bounce time derivative, which is eigenfunction of the quantum fluctuations Sturm-Liouville operator with zero eigenvalue (due to time translational invariance), is not the ground state. There must be a negative eigenvalue [12] in the fluctuation spectrum causing an imaginary contribution to the total partition function. The decay rate of the false vacuum arises precisely from this negative eigenvalue which, moreover, is unique as the bounce path has one node along the time axis.

While the general formalism of metastability is settled at least in one dimension and

in the semiclassical method [13, 14], the evaluations of $\Gamma$ for specific systems are generally 
based on the Langer-Coleman approach [15] which assumes a system with infinite size $L$ and therefore, strictly speaking, holds in the zero temperature limit of the quantum field theory once $L$ is taken equal to $\hbar c / K_{B} T$. The same formal approach can be used in classical systems to calculate the noise-induced transition rate between locally stable states, for example the domain reversal rate in micromagnets [16] and the instabilities in metallic nanowires [17]. In such systems however the character of the transition between activation regimes crucially depends on the finite spatial extent [18], thus quantitative estimates of the Kramer escape rate require an extension of the Langer-Coleman method to the more complex finite size case. In quantum tunneling systems, the counterpart of the latter is represented by the finite $T$ quantum regime below the crossover temperature $T_{c}^{*}$ at which thermal activated processes set in. Below $T_{c}^{*}$, the decay rate stems from purely quantum fluctuations effects and the $\Gamma$ dependence on $T$ is governed by the softening of the fluctuation spectrum. To determine this phenomenon with accuracy one has to face the non trivial task of computing fluctuation functional determinants at low but finite $T$ [19] which also includes a knowledge of the negative eigenvalue in the spectrum. This paper focuses on this issues developing the finite temperature theory of metastability below $T_{c}^{*}$ for the case of a model cubic potential. In particular, the softening of the fluctuations is analysed quantitatively by means of the Jacobi elliptic functions formalism [20] which has the advantage to monitor the evolution of the system at any $T$, free from approximations regarding the form of the bounce and its fluctuation spectrum. The explicit equations which determine such softening are provided. Activation effects [21, 22] are not discussed in this work which assumes a closed system. Metastability in the presence of dissipation has been studied in Ref.[23].

As in the space-time Euclidean path integral the time is an inverse temperature, the zero $T$ theory assumes that the bounce classical motion lasts an infinite time whereas the finite $T$ theory realistically admits that the excursion time for the bounce may be finite. This consideration permits to derive the family of energy dependent paths which solve the classical equation of motion as shown in Section 2 for the cubic potential. In Section 3, using the theory of the functional determinants, I determine analytically and compute the quantum fluctuations contribution to the path integral. The solution of the Lamè equation which governs the fluctuation spectrum is presented together with the evaluation of the temperature effects on the lowest lying eigenmodes and their eigenvalues. Section 4 contains the results for the decay rate of the metastable cubic potential and a comparison with the 
case of the metastable quartic potential. Some final remarks are made in Section 5.

\section{Cubic Potential Model}

Consider a particle of mass $M$ in the one dimensional cubic potential:

$$
V(x)=\frac{M \omega^{2}}{2} x^{2}-\frac{\gamma}{3} x^{3}
$$

plotted in Fig. 1(a) for several energies $\hbar \omega$. Say a the position of the top of barrier whose height is $V(a)=\gamma a^{3} / 6$ with $\gamma=M \omega^{2} / a$. I take throughout the paper, $a=1 \AA$ and $M=10^{4} m_{e}$ with $m_{e}$ being the electron mass. At $x=0$ the particle is in a local minimum,

see Fig. 1(a), from which it cannot escape classically and, in the real time formalism, only $x_{c l}=0$ solves the classical equation of motion. Such local minimum is however metastable as quantum fluctuations allow the particle to explore the abyss at $x \geq 3 a / 2$. Let's see how.

\section{A. Classical Path}

A non trivial classical solution can be found in the Euclidean space. In fact, performing a Wick rotation from the real to the imaginary time, $t \rightarrow-i \tau$, the equation of motion reads:

$$
M \ddot{x}_{c l}(\tau)=V^{\prime}\left(x_{c l}(\tau)\right)
$$

where $V^{\prime}$ means derivative with respect to $x_{c l}$. The particle path $x(\tau)$ has been split in the sum of a classical and a quantum component, $x(\tau)=x_{c l}(\tau)+\eta(\tau)$. The Wick rotation is equivalent to turn the potential upside down with respect to the real time as shown in Fig. 1(b) for $\hbar \omega=10 \mathrm{meV}$. In the reversed potential the classical motion can take place.

Integrating Eq. (3), one gets:

$$
\frac{M}{2} \dot{x}_{c l}^{2}(\tau)-V\left(x_{c l}(\tau)\right)=E
$$

with the constant $E$ representing the classical energy. Defining: 


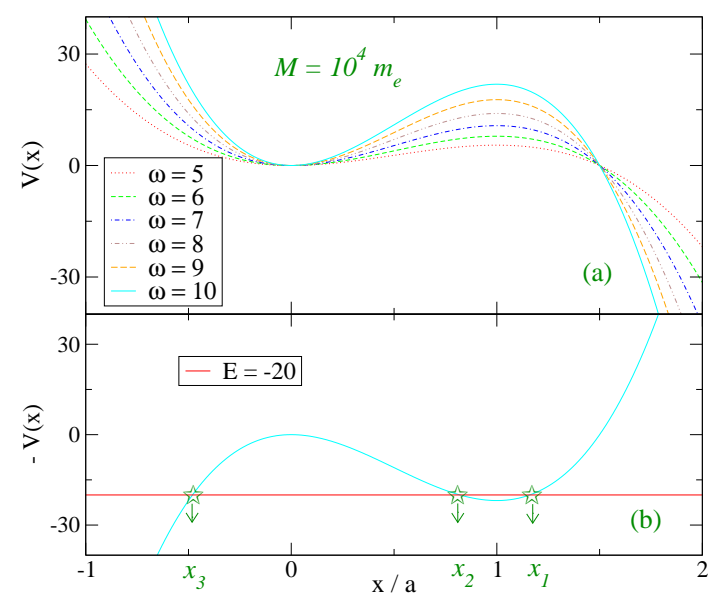

FIG. 1: (Color online) (a) Cubic potential (in $m e V$ ) for several oscillator energies, (b) Cubic potential for $\hbar \omega=10 \mathrm{meV}$ in the imaginary time representation. The intersections with the constant energy $E$ (in $m e V$ ) define the turning points for the classical motion.

$$
\begin{aligned}
& \chi_{c l}(\tau)=\frac{2}{3} \frac{x_{c l}(\tau)}{a} \\
& \kappa=\frac{4 E}{27 V(a)}
\end{aligned}
$$

Eq. (4) is easily integrated to yield:

$$
\tau-\tau_{0}= \pm \frac{1}{\omega} \int_{\chi_{c l}\left(\tau_{0}\right)}^{\chi_{c l}(\tau)} \frac{d \chi}{\sqrt{-\chi^{3}+\chi^{2}+\kappa}}
$$

where $\tau_{0}$ is the center of motion between the turning points at which the path velocity vanishes. Thus the physical picture set by the boundary conditions is that, see Fig. 11(b), of a particle starting from $x_{2}$ at the time $\tau=-L / 2$, reaching $x_{1}$ at $\tau=\tau_{0}$ and returning to the initial position at $\tau=L / 2$. Then, Eq. (6) has a time reversal invariant solution whose period $L$ is finite and dependent on $E$. Phase transitions phenomena in spatially extended systems are analysed by formally similar models with $L$ being the size of the system [18, 24]. Looking at Fig. 1(b), one captures qualitatively that the amplitude $x_{1}-x_{2}$ attains the largest value for the $E=0$ motion while $x_{2}$ and $x_{3}$ coincide. $x_{3}$ becomes negative for $E<0$ motions.

The turning points $x_{1}, x_{2}, x_{3}$ are given by the zeros of the equation $-\chi^{3}+\chi^{2}+\kappa=0(\chi \equiv$ $2 x /(3 a))$ which admits three real solutions for $\kappa \in[-4 / 27,0]$, that is for $E \in[-V(a), 0]$. After some algebra I find: 


$$
\begin{aligned}
& \chi_{1}=\frac{1}{3}+\frac{2}{3} \cos (\vartheta) \\
& \chi_{2}=\frac{1}{3}+\frac{2}{3} \cos (\vartheta-2 \pi / 3) \\
& \chi_{3}=\frac{1}{3}+\frac{2}{3} \cos (\vartheta-4 \pi / 3) \\
& \vartheta=\frac{1}{3} \arccos \left(\frac{27 \kappa}{2}+1\right)
\end{aligned}
$$

At the bounds of the energy range, Eq. (7) yields:

$$
\begin{aligned}
& \mathbf{E}=\mathbf{0} \Rightarrow \chi_{1}=1 ; \chi_{2}=\chi_{3}=0 \\
& \mathbf{E}=-\mathbf{V}(\mathbf{a}) \Rightarrow \chi_{1}=\chi_{2}=2 / 3 ; \chi_{3}=-1 / 3
\end{aligned}
$$

Thus, at the sphaleron energy $E_{s p h}=|E|=V(a)$ [25], the amplitude of the finite time solution has to shrink into a point. Let's find the general solution of Eq. (6) by pinning the center of motion at $\chi_{c l}\left(\tau_{0}\right)=\chi_{1}$ and using the result [26]:

$$
\begin{aligned}
& \int_{\chi_{c l}(\tau)}^{\chi_{1}} \frac{d \chi}{\sqrt{\left(\chi_{1}-\chi\right)\left(\chi-\chi_{2}\right)\left(\chi-\chi_{3}\right)}}=\frac{2 F(\lambda, p)}{\sqrt{\chi_{1}-\chi_{3}}} \\
& \lambda=\arcsin \left(\sqrt{\frac{\chi_{1}-\chi_{c l}(\tau)}{\chi_{1}-\chi_{2}}}\right) \\
& p=\sqrt{\frac{\chi_{1}-\chi_{2}}{\chi_{1}-\chi_{3}}}
\end{aligned}
$$

where $F(\lambda, p)$ is the elliptic integral of the first kind with amplitude $\lambda$ and modulus $p$. Then, through Eqs. (5), (6) , (9), I derive the bounce solution of the finite time theory:

$$
\begin{aligned}
& x_{c l}(\tau)=\frac{3 a}{2}\left[\chi_{1} c n^{2}(\varpi, p)+\chi_{2} s n^{2}(\varpi, p)\right] \\
& \varpi=\sqrt{\chi_{1}-\chi_{3}} \frac{\omega}{2}\left(\tau-\tau_{0}\right)
\end{aligned}
$$

$\operatorname{sn}(\varpi, p)$ and $c n(\varpi, p)$ are the sine- and cosine- amplitudes respectively [20]. The modulus $p$ incorporates the classical mechanics of the problem through the second of Eq. (5) and Eq. (7). At $\mathrm{E}=0, p=1$, the bounce of the infinite time theory is recovered: 


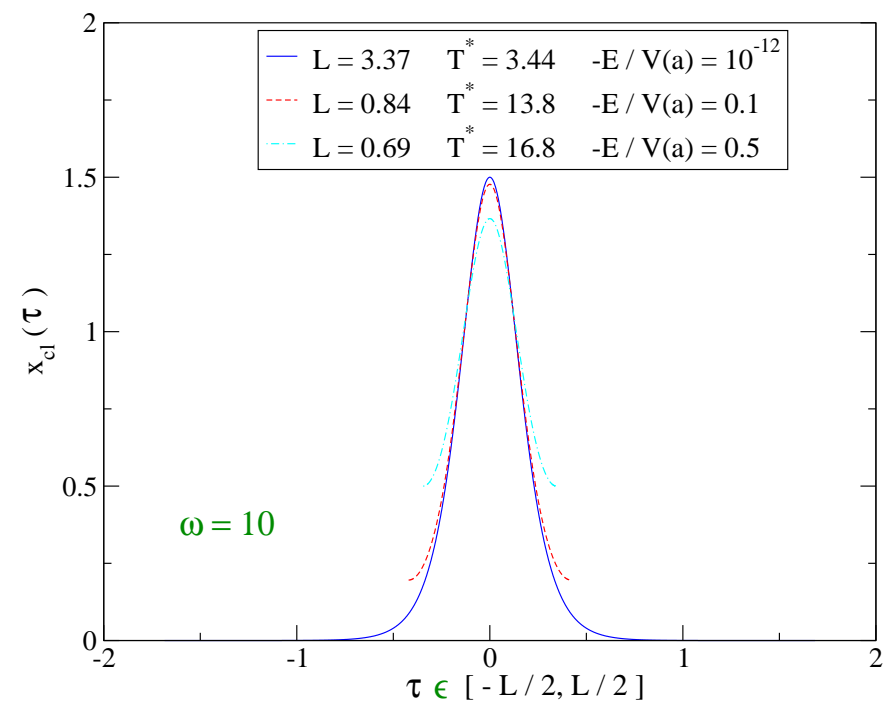

FIG. 2: (Color online) Shape of the bounce solution for three classical energies $E$. The oscillation period $L / \hbar$ is in units $m e V^{-1}$ and $T^{*}$ in $K$.

$$
x_{c l}(\tau)=\frac{3 a}{2} \operatorname{cn}^{2}(\varpi, 1)=\frac{3 a}{2} \operatorname{sech}^{2}\left(\frac{\omega}{2}\left(\tau-\tau_{0}\right)\right)
$$

At the sphaleron energy, $p=0$, the bounce solution is in fact a point-like object set at the bottom of the valley in the reversed potential: $x_{c l}(\tau)=a$. Thus, Eq. (10) defines the transition state which is a saddle for the action below the sphaleron. Representing the Jacobi elliptic functions in Eq. (10) through trigonometric series [26] suitable to computation, I plot the classical path in Fig. 2 which makes evident how the bounce amplitude contracts by increasing the energy over potential height ratio (in absolute value). While the bounce can be physically interpreted as a pair of domain walls, it is clear that the extent of the $\tau$-region over which the domain walls separate the turning points continuously shrinks by approaching the crossover. The center of motion $\tau_{0}$ has been set equal to zero with no loss of generality being the system invariant for time translation.

As the bounce is a combination of squared Jacobi elliptic functions, its period is $2 K(p)$ with $K(p)=F(\pi / 2, p)$ being the complete elliptic integral of the first kind [20]. Hence, from Eq. (10), I get: 


$$
\sqrt{\chi_{1}-\chi_{3}} \frac{\omega}{4} L=K(p)
$$

which establishes the relation between the oscillation period and the classical energy embedded in the turning points. As in the Matsubara formalism, one can map the imaginary time onto the temperature axis, $L=\hbar /\left(K_{B} T^{*}\right)$, where $T^{*}$ is the temperature at which the particle makes the journey to and from the edge of the abyss for a given $E$. Then, only periodic bounces whose period equals the inverse temperature determine the decay rate and the finite time theory can be viewed as a finite $T^{*}$ theory. From Eq. (12) I get:

$$
K_{B} T^{*}=\frac{\hbar \omega}{4} \frac{\sqrt{\chi_{1}-\chi_{3}}}{K(p)}
$$

The $L$ and $T^{*}$ values given in Fig. 2 are computed on the base of Eqs. (12, (13) respectively.

At the sphaleron, Eq. (13) yields

$$
K_{B} T_{c}^{*}=\frac{\hbar \omega}{2 \pi}
$$

that marks the transition temperature between quantum and activated regimes. Such value represents the upper bound for the validity of the model and precisely sets the Goldanskii criterion [27] for the cubic potential. Taking $\hbar \omega=10 \mathrm{meV}$, the following calculations are carried out in the low temperature range up to $T_{c}^{*}=18.469 \mathrm{~K}$.

\section{B. Classical Action}

The classical action $A\left[x_{c l}\right]$ for the bounce in the finite temperature theory can be computed either in terms of the path velocity $\dot{x}_{c l}(\tau)$ :

$$
\begin{aligned}
& A\left[x_{c l}\right]=2 M \int_{-L / 2}^{\tau_{0}} d \tau\left[\dot{x}_{c l}(\tau)\right]^{2}-E \cdot L(E) \\
& \dot{x}_{c l}(\tau)=\frac{3 a}{2} \mathcal{F} \cdot \operatorname{sn}(\varpi, p) c n(\varpi, p) d n(\varpi, p) \\
& \mathcal{F}=-\omega\left(\chi_{1}-\chi_{2}\right) \sqrt{\chi_{1}-\chi_{3}}
\end{aligned}
$$


$\left(d n(\varpi, p)\right.$ is the delta- amplitude [20]) or, in terms of the potential $V\left(x_{c l}\right)$ :

$$
A\left[x_{c l}\right]=\frac{27 V(a)}{\omega} \int_{\chi_{2}}^{\chi_{1}} d \chi \sqrt{\kappa+\chi^{2}-\chi^{3}}-E \cdot L(E)
$$

Both ways require computation of $L(E)$ through Eqs. (15), (17), (12).

In the $E \rightarrow 0$ limit, I get the result

$$
\frac{A\left[x_{c l}\right]}{\hbar} \rightarrow \frac{6 M^{3} \omega^{5}}{5 \hbar \gamma^{2}}
$$

which serves as testbench for the computational method. The inverse dependence on the anharmonic force constant $\gamma$ reflects the well known fact that metastable systems are intrinsecally non perturbative and provides the fundamental motivation for the semiclassical treatment. Eq. (17) permits to set the potential parameters such as the condition $A\left[x_{c l}\right] \gg \hbar$ holds and the semiclassical method is thus justified. As $M$ and $a$ have been taken constant, $\frac{A\left[x_{c l}\right]}{\hbar} \propto \omega$ in the $E \rightarrow 0$ limit. The bounce velocity and the classical action are displayed in Fig. 3 and Fig. 4 respectively. Note that $A\left[x_{c l}\right]$ decreases smoothly with increasing temperature and absolute value of energy (inset (a) in Fig. 4) suggesting that the transition to the activated regime above $T_{c}^{*}$ is of second order [12, 28]. Although I'm dealing here with a closed system, it is worth emphasizing that the inclusion of a dissipative environment [29] would decrease $T_{c}^{*}$ but would not change the character of the crossover.

In general, both first and second order transitions have been found in tunneling systems depending on the shape of the potential [30, 31, 32]. The criterion used to establish the order of the transitions in periodic tunneling systems has been formulated by Chudnovsky [33] on the base of the behavior of $L(E)$ : a monotonic dependence of the period below the sphaleron points to a smooth crossover whereas a nonmonotonic $L(E)$ indicates a sharp transition. The inset (b) in Fig. 4 clearly shows that the former case applies to the cubic potential in Eq. (2). $L(E)$ decreases monotonically and, consistently, the action versus $T^{*}$ is convex upwards. At the sphaleron, I find numerically: $L\left(E_{s p h}\right) / \hbar=0.628 \mathrm{meV}^{-1}$. Note that such value corresponds to $2 \pi / \hbar \omega[34]$ and this is not by accident. In fact at the sphaleron the bounce is a point, that is a static solution of Eq. (4) but, near the sphaleron, the periodic path is the sum of the sphaleron and an oscillation with negative eigenvalue $\varepsilon_{-1}$ whose period 


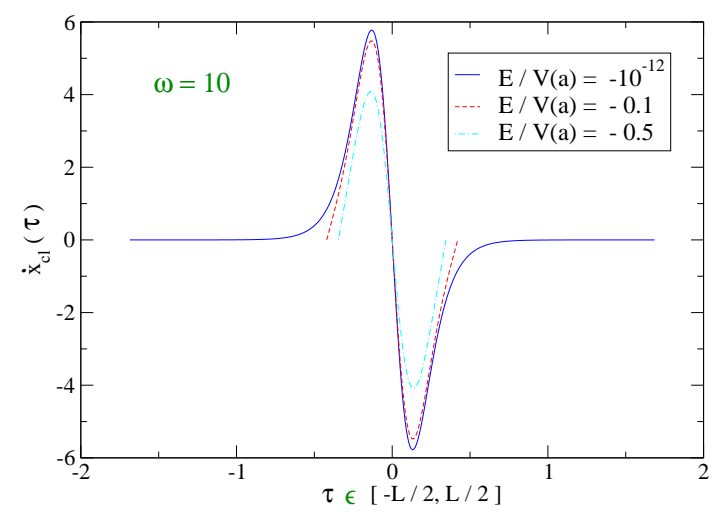

FIG. 3: (Color online) Bounce velocity versus imaginary time for the same parameters as in Fig. 2.

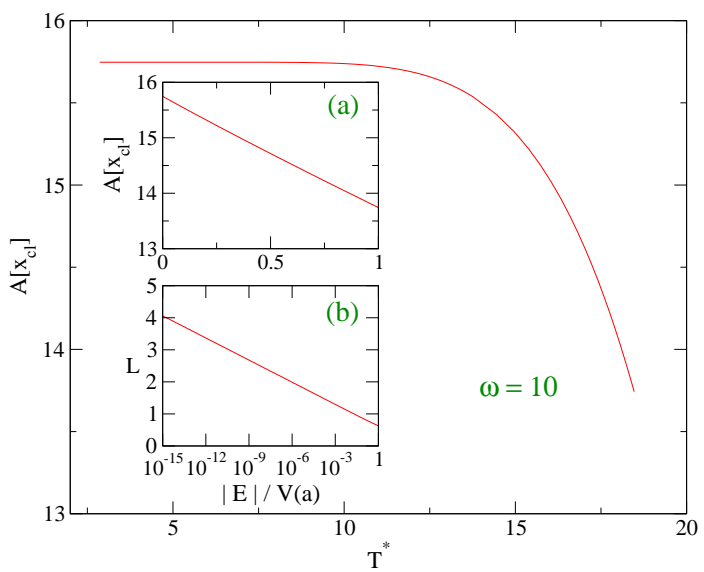

FIG. 4: (Color online) Classical action (in units $\hbar$ ) versus temperature. Insets: (a) Classical Action versus Energy over Barrier potential height ratio on a linear scale; (b) Oscillation period (over $\hbar$ ) versus Energy over Barrier potential height ratio on a log scale.

tends to $L\left(E_{s p h}\right)=2 \pi / \sqrt{\left|\varepsilon_{-1}\right|}[35]$. Then one infers that, for $|E| \rightarrow E_{s p h}, \varepsilon_{-1} \rightarrow-\omega^{2}$. I'll come back to this point towards the end of the next Section.

\section{Quantum Fluctuations}

Let's expand the action around the classical path up to second order in the fluctuations 


$$
\begin{aligned}
& A[x] \sim A\left[x_{c l}\right]+\delta A[\eta]+\frac{1}{2 !} \delta^{2} A[\eta] \\
& \delta A[\eta]=\int_{-L / 2}^{L / 2} d \tau\left(M \dot{x}_{c l}(\tau) \dot{\eta}(\tau)+V^{\prime}\left(x_{c l}(\tau)\right) \eta(\tau)\right) \\
& \delta^{2} A[\eta] \equiv 2 A_{f}[\eta] \\
& A_{f}[\eta]=\int_{-L / 2}^{L / 2} d \tau\left(\frac{M}{2} \dot{\eta}^{2}(\tau)+\frac{1}{2} V^{\prime \prime}\left(x_{c l}(\tau)\right) \eta^{2}(\tau)\right)
\end{aligned}
$$

Being the classical solution an extremum for the action, the first functional derivative vanishes and, after partial integration in the first term of $\delta A(\eta)$, one gets the Euler-Lagrange Eq. (3).

Further, differentiating Eq. (3) with respect to $\tau$, one observes that $\dot{x}_{c l}(\tau)$ solves the homogeneous equation associated to the second order Schrödinger-like differential operator in $A_{f}[\eta]$

$$
\begin{aligned}
& \hat{O} \eta_{n}(\tau)=\varepsilon_{n} \eta_{n}(\tau) \\
& \hat{O} \equiv-\partial_{\tau}^{2}+V^{\prime \prime}\left(x_{c l}(\tau)\right) / M \\
& \eta(\tau)=\sum_{n=-1}^{\infty} \varsigma_{n} \eta_{n}(\tau)
\end{aligned}
$$

where the $\varepsilon_{n}$ are the fluctuations eigenvalues while the coefficients $\varsigma_{n}$ of the series expansion in ortonormal components $\eta_{n}(\tau)$ define the measure of the fluctuation paths integration. Then, as a general consequence of the $\tau$-translational invariance, $\dot{x}_{c l}(\tau)$ is proportional to the eigenmode $\eta_{0}(\tau)$ with $\varepsilon_{0}=0$. The latter cannot be the ground state as, see Fig. [3, $\dot{x}_{c l}(\tau)$ has one node hence, the quantum fluctuation ground state has negative eigenvalue. From Eq. (15)), note that for any two points $\varpi_{1}, \varpi_{2}$ such that $\varpi_{2}=\varpi_{1} \pm 2 K(p), \dot{x}_{c l}\left(\varpi_{2}\right)=\dot{x}_{c l}\left(\varpi_{1}\right)$. The important consequence is that the fluctuation eigenmodes obey periodic boundary conditions (PBC).

After these observations we are ready to calculate the space-time Euclidean path integral between the positions $x_{i}$ and $x_{f}$ connected in the time $L$. In the semiclassical model, it is given by 


$$
\begin{aligned}
& <x_{f} \mid x_{i}>_{L}=\exp \left[-\frac{A\left[x_{c l}\right]}{\hbar}\right] \cdot \int D \eta \exp \left[-\frac{\left.A_{f}[\eta]\right]}{\hbar}\right] \\
& \int D \eta=\aleph \prod_{n=-1}^{\infty} \int_{-\infty}^{\infty} \frac{d \varsigma_{n}}{\sqrt{2 \pi \hbar / M}}
\end{aligned}
$$

$\aleph$ depends only on the functional integral measure. $x_{i}$ and $x_{f}$ coincide for the periodic bounce, thus Eq. (20) represents the single bounce contribution $Z_{1}$ to the total partition function $Z_{T}$. The latter also contains the effects of all multiple (non interacting) excursions to and from the abyss which is equivalent to sum [7] over an infinite number of single bounce contributions like $Z_{1}$. Moreover, also the static solution of Eq. (33),$x_{c l}=0$ contributes with the harmonic partition function $Z_{h}$ which can be easily determined using the measure in Eq. (20). Summing up, $Z_{T}$ is given by

$$
\begin{aligned}
& Z_{T}=Z_{h} \exp \left(Z_{1} / Z_{h}\right) \\
& Z_{h}=\aleph|\operatorname{Det}[\hat{h}]|^{-1 / 2}
\end{aligned}
$$

$\operatorname{Det}[\hat{h}]$ being the harmonic fluctuation determinant. Being the decay rate $\Gamma$ proportional to the imaginary exponential argument through the Feynman-Kac formula [7], it follows that there is no need to determine $\aleph$ as it cancels out in the ratio $Z_{1} / Z_{h}$.

Let's proceed to evaluate the fluctuation term in $Z_{1}$ which, formally, is worked out by carrying out Gaussian path integrals yielding:

$$
\begin{aligned}
& \int \operatorname{D\eta } \exp \left[-\frac{A_{f}[\eta]}{\hbar}\right]=\aleph \cdot \operatorname{Det}[\hat{O}]^{-1 / 2} \\
& \operatorname{Det}[\hat{O}] \equiv \prod_{n=-1}^{\infty} \varepsilon_{n}
\end{aligned}
$$

Eq. (22) is however divergent due to the Goldstone mode which reflects the fact that $\tau_{0}$, the center of the bounce, can be located arbitrarily inside $L$. However such mode can be treated separately [28]: the divergent integral over the coordinate $d \varsigma_{0}$ associated to the zero mode in the measure $D \eta$ is transformed into a $d \tau_{0}$ integral. Accordingly the eigenvalue $\varepsilon_{0}=0$ is extracted from $\operatorname{Det}[\hat{O}]$ and its contribution to Eq. (22) is replaced as follows 


$$
\begin{aligned}
& \left(\varepsilon_{0}\right)^{-1 / 2} \rightarrow \sqrt{\frac{M}{2 \pi \hbar}} \bar{N}^{-1} L \\
& \bar{N}^{-1}=\sqrt{2 \int_{0}^{L / 2} d \tau\left|\dot{x}_{c l}(\tau)\right|^{2}}
\end{aligned}
$$

To be rigorous, this replacement holds in the approximation of quadratic fluctuations [15] while higher order terms may be significant around the crossover. It is also worth noticing that Eq. (23) is often encountered in the form $\left(\varepsilon_{0}\right)^{-1 / 2} \rightarrow \sqrt{\frac{A\left[x_{c l} l\right.}{2 \pi \hbar}} L$. However, this is correct only in the zero $T$ limit where $A\left[x_{c l}\right]$ equals $M \bar{N}^{-2}$ (see Eq. (15)) while, approaching $T_{c}^{*}$, the difference between the two objects gets large. Exactly at $T_{c}^{*}$, the norm of the bounce velocity vanishes while the action is finite. This fact is crucial in establishing the behavior of the decay rate at the crossover as shown below.

Now I tackle the problem of the evaluation of the regularized determinant $D e t^{R}[\hat{O}]$ defined by $\operatorname{Det}[\hat{O}]=\varepsilon_{0} \cdot \operatorname{Det}^{R}[\hat{O}]$.

\section{A. Fluctuation Determinant}

The calculation of $\operatorname{Det}^{R}[\hat{O}]$ requires knowledge only of the classical paths. This is a fundamental feature of the theory of functional determinants of second order differential operators first developed for Dirichlet boundary conditions [8] and then extended to general operators and boundary conditions in several ways [36, 37]. As shown above the path velocity obeys $\mathrm{PBC}$ for any two points $\varpi_{1}, \varpi_{2}$ separated by the period $2 K(p)$. The latter corresponds to the oscillation period $L$ along the $\tau$-axis. It can be easily checked that also the path acceleration fulfills the PBC. Then, the regularized determinant is given by

$$
\operatorname{Det}^{R}[\hat{O}]=\frac{<f_{0} \mid f_{0}>\left(f_{1}\left(\varpi_{2}\right)-f_{1}\left(\varpi_{1}\right)\right)}{f_{0}\left(\varpi_{1}\right) W\left(f_{0}, f_{1}\right)}
$$

where $f_{0}, f_{1}$ are two independent solutions of the homogeneous equation: $\hat{O} \eta_{n}(\tau)=0$. $W\left(f_{0}, f_{1}\right)$ is their Wronskian and $<f_{0} \mid f_{0}>$ is the squared norm. $f_{0}$ is obviously $\dot{x}_{c l}$ while $f_{1}$ can be taken as

$$
f_{1}=\frac{\partial x_{c l}}{\partial q} ; q \equiv p^{2}
$$


Using Eq. (10), derivatives and properties of the elliptic functions, I derive for $f_{1}$ :

$$
\begin{aligned}
f_{1}= & \frac{3 a}{2}\left[\frac{\partial \chi_{1}}{\partial q} c n^{2}(\varpi, p)+\frac{\partial \chi_{2}}{\partial q} s n^{2}(\varpi, p)\right. \\
& \left.-2\left(\chi_{1}-\chi_{2}\right) \operatorname{sn}(\varpi, p) c n(\varpi, p) d n(\varpi, p) \cdot \frac{\partial F(\lambda, p)}{\partial q}\right] \\
\frac{\partial \chi_{1}}{\partial q}= & \frac{1-p^{2}}{2\left(1-p^{2}+p^{4}\right)^{3 / 2}} \\
\frac{\partial \chi_{2}}{\partial q}= & \frac{-1}{2\left(1-p^{2}+p^{4}\right)^{3 / 2}} \\
\frac{\partial F(\lambda, p)}{\partial q}= & \frac{1}{2 \bar{p}^{2}}\left[\frac{E(\lambda, p)-\bar{p}^{2} F(\lambda, p)}{p^{2}}-\frac{\sin \lambda \cos \lambda}{\sqrt{1-p^{2} \sin ^{2} \lambda}}\right] \\
& \bar{p}^{2}=1-p^{2}
\end{aligned}
$$

where $E(\lambda, p)$ is the elliptic integral of the second kind.

The Wronskian, being constant along $\tau$, can be calculated in any convenient point. Let's take $\tau_{0}$ as $f_{0}\left(\tau_{0}\right)=0$. Then:

$$
\begin{aligned}
& \left.W\left(f_{0}, f_{1}\right)\right|_{\tau_{0}}=-\dot{f}_{0}\left(\tau_{0}\right) f_{1}\left(\tau_{0}\right) \\
& =\frac{9}{8} a^{2} \omega^{2}\left(\chi_{1}-\chi_{2}\right)\left(\chi_{1}-\chi_{3}\right) \frac{\partial \chi_{1}}{\partial q}
\end{aligned}
$$

and $<f_{0} \mid f_{0}>\equiv \bar{N}^{-2}$ given in Eq. (23) $)$.

Thus, all ingredients are known to calculate $\operatorname{Det}^{R}[\hat{O}]$ : evaluating Eq. (26) on the $\tau-$ range boundaries, Eq. (24) transforms into

$$
\operatorname{Det}^{R}[\hat{O}]=\frac{2}{\omega \sqrt{\chi_{1}-\chi_{3}} \bar{p}^{2}}\left[\frac{E(\pi / 2, p)-\bar{p}^{2} K(p)}{p^{2}}\right] \cdot \frac{<f_{0} \mid f_{0}>}{W\left(f_{0}, f_{1}\right)}
$$

which can be computed using Eqs. (23), (27). It is however known in the theory of functional determinants [8] that only ratios of determinants are meaningful in value and sign, such ratios arising naturally in the path integral method as it has been pointed out above. In fact, $\operatorname{Det}^{R}[\hat{O}]$ would diverge in the $T^{*} \rightarrow 0$ limit due to the fact that the determinant is the product over an infinite number of eigenvalues with magnitude greater than one. 
Consistently with Eq. (21), $\operatorname{Det}^{R}[\hat{O}]$ has to be normalized over $\operatorname{Det}[\hat{h}]\left(\hat{h} \equiv-\partial_{\tau}^{2}+\omega^{2}\right)$ which, in the case of PBC, is: $\operatorname{Det}[\hat{h}]=-4 \sinh ^{2}(\omega L / 2)$. The normalization cancels the exponential divergence and makes the ratio finite.

Then, observing that for $E \rightarrow 0\left(T^{*} \rightarrow 0\right)$ :

$$
\begin{aligned}
& \left.W\left(f_{0}, f_{1}\right)\right|_{\tau_{0}} \rightarrow \frac{9}{8} a^{2} \omega^{2}\left(1-p^{2}\right) \\
& <f_{0} \mid f_{0}>\rightarrow \frac{6}{5} a^{2} \omega \\
& K(p) \rightarrow \ln \left(4 / \sqrt{1-p^{2}}\right)
\end{aligned}
$$

from Eq. (28), I finally get the finite ratio

$$
\frac{\operatorname{Det}^{R}[\hat{O}]}{\operatorname{Det}[\hat{h}]} \rightarrow-\frac{1}{60 \omega^{2}}
$$

The dimensionality $\left[\omega^{-2}\right]$ correctly accounts for the fact that one eigenvalue has been extracted from $\operatorname{Det}^{R}[\hat{O}]$. The computation of Eq. (28) becomes rather time consuming in the $T^{*} \rightarrow 0$ limit where elliptic integrals need to be evaluated with high accuracy in order to get the correct exponential divergence.

The plot of $\operatorname{Det}^{R}[\hat{O}]$ versus temperature is shown in Fig. 5 up to $T^{*}=18.339 K<T_{c}^{*}$ while the inset displays the computed inverse ratio normalized over $-60 \omega^{2}$ : the $T^{*} \rightarrow 0$ limit is in fact an excellent estimate up to $T^{*} \sim 10 \mathrm{~K}$ whereas a strong deviation is found at larger $T^{*}$ up to $\sim T_{c}^{*}$. Exactly at $T_{c}^{*}, \operatorname{Det}^{R}[\hat{O}]$ vanishes thus causing the divergence of the inverse ratio. This divergence may look surprising since the zero mode had been extracted from the determinant: as it will be unravelled in the next Subsection, there must be a quantum fluctuation mode which softens by increasing $T^{*}$ and ultimately vanishes at the sphaleron.

I want to emphasize the key role played by the bounce velocity norm in getting $\operatorname{Det}^{R}[\hat{O}] \sim$ 0 at $T^{*} \sim T_{c}^{*}$ : close to the sphaleron, $<f_{0} \mid f_{0}>\propto p^{4}$ and $W\left(f_{0}, f_{1}\right) \propto p^{2}$ thus the squared norm drops to zero faster than any other term in Eq. (28). This feature is made evident in Fig. [6 where the plots of $\operatorname{Det}^{R}[\hat{O}] /<f_{0} \mid f_{0}>$ and the squared norm are given separately against temperature up to $T_{c}^{*}$.

Eventually, note that the determinants ratio is negative and this sign has physical meaning as it is due precisely to the negative ground state of the fluctuation spectrum. As $Z_{1} / Z_{h}$ 


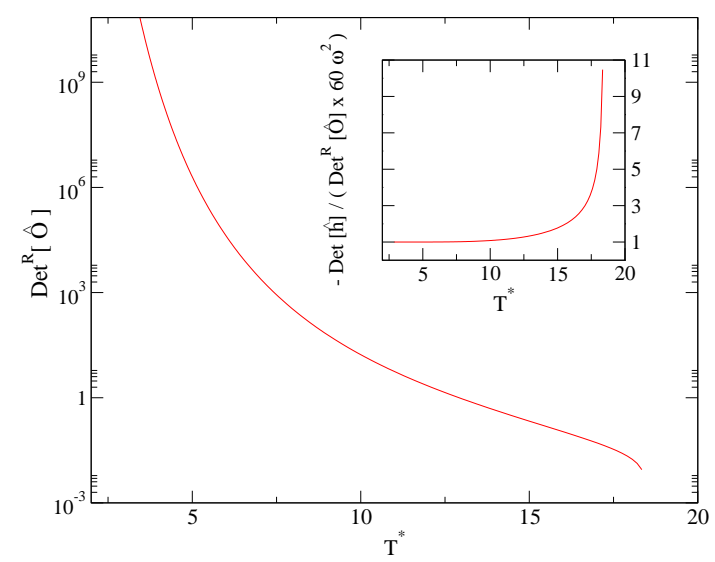

FIG. 5: (Color online) Regularized fluctuation determinant versus temperature up to $T^{*}=$ $18.339 \mathrm{~K}$. The inset shows the ratio between harmonic oscillator and regularized fluctuation determinants. Such ratio is normalized over the characteristic value $-60 \omega^{2}$ found in the zero temperature theory.
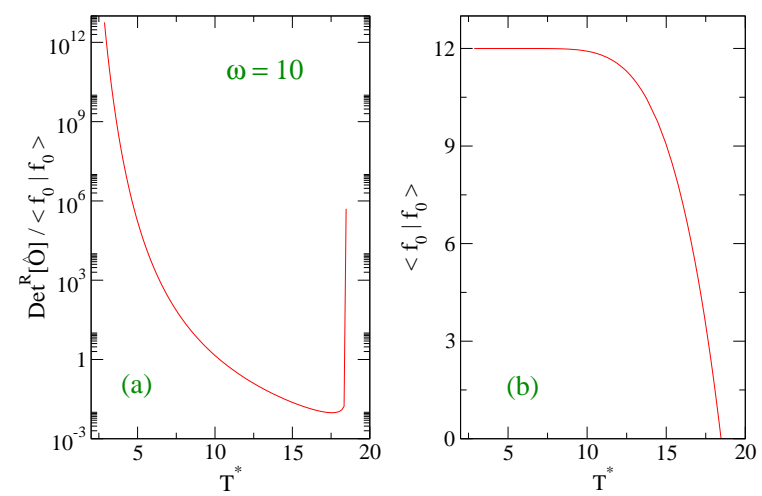

FIG. 6: (Color online) (a) Regularized fluctuation determinant over norm of the zero eigenmode; (b) norm of the zero eigenmode. Both curves are plotted versus temperature including the crossover point.

contributes to the partition function by the square root of the fluctuation determinants (inverse) ratio it follows that such contribution is purely imaginary. To weigh the effect of the negative eigenvalue $\varepsilon_{-1}$, its analytical expression has to be determined at finite $T^{*}$. This is done in the next Subsection.

\section{B. Lamè Equation}

Let's take Eq. (19) with the second derivative of the potential given by 


$$
V^{\prime \prime}\left(x_{c l}(\tau)\right)=M \omega^{2}\left(1-\frac{2}{a} x_{c l}(\tau)\right)
$$

Then, using Eq. (10) and working out the algebra, I get the stability equation which governs the fluctuation spectrum

$$
\begin{aligned}
& \frac{d^{2}}{d \varpi^{2}} \eta_{n}(\tau)=\left[12 p^{2} s n^{2}(\varpi, p)+\mathcal{A}_{n}\right] \eta_{n}(\tau) \\
& \mathcal{A}_{n}=\frac{4\left(1-3 \chi_{1}\right)}{\chi_{1}-\chi_{3}}-\frac{4 \varepsilon_{n}}{\omega^{2}\left(\chi_{1}-\chi_{3}\right)} \\
& 12 \equiv l(l+1)
\end{aligned}
$$

This is the Lamè equation in the Jacobian form for the case $l=3[20]$. For a given $l$ and $p$, Eq. (32) yields periodic solutions (which can be expanded in infinite series) for an infinite sequence of characteristic $\mathcal{A}_{n}$ values. The continuum of the fluctuation spectrum stems from this sequence. However, being $l$ positive and integer, the first $2 l+1$ solutions of Eq. (32) are not infinite series but polynomials in the Jacobi elliptic functions with real period $2 K(p)$ or $4 K(p)$. Being the period of the potential [38], $2 K(p)$ plays the role of a lattice constant.

Then, Eq. (32) admits 7 polynomial solutions with eigenvalues $\mathcal{A}_{n}, n \in[-l, l]$, from which the corresponding $\varepsilon_{n}$ are derived [39]:

$$
\begin{aligned}
& \varepsilon_{3}=\omega^{2}\left(\alpha_{1}+\alpha_{2}\left[5\left(1+p^{2}\right)+2 \sqrt{4 p^{4}-7 p^{2}+4}\right]\right) \\
& \varepsilon_{-3}=\omega^{2}\left(\alpha_{1}+\alpha_{2}\left[5\left(1+p^{2}\right)-2 \sqrt{4 p^{4}-7 p^{2}+4}\right]\right) \\
& \varepsilon_{2}=\omega^{2}\left(\alpha_{1}+\alpha_{2}\left[5+2 p^{2}+2 \sqrt{p^{4}-p^{2}+4}\right]\right) \\
& \varepsilon_{-2}=\omega^{2}\left(\alpha_{1}+\alpha_{2}\left[5+2 p^{2}-2 \sqrt{p^{4}-p^{2}+4}\right]\right) \\
& \varepsilon_{1}=\omega^{2}\left(\alpha_{1}+\alpha_{2}\left[2+5 p^{2}+2 \sqrt{4 p^{4}-p^{2}+1}\right]\right) \\
& \varepsilon_{-1}=\omega^{2}\left(\alpha_{1}+\alpha_{2}\left[2+5 p^{2}-2 \sqrt{4 p^{4}-p^{2}+1}\right]\right) \\
& \varepsilon_{0}=0 \\
& \alpha_{1} \equiv 1-3 \chi_{1} \\
& \alpha_{2} \equiv \frac{\chi_{1}-\chi_{3}}{4}
\end{aligned}
$$



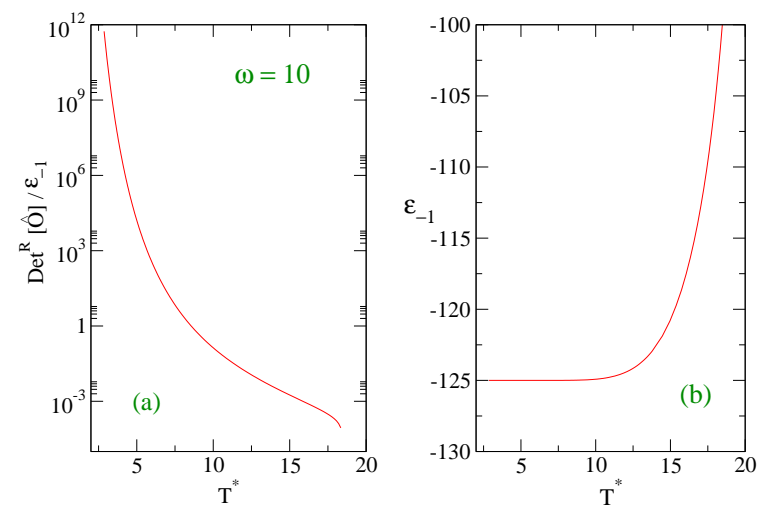

FIG. 7: (Color online) (a) Absolute value of regularized fluctuation determinant over ground state eigenvalue ratio (units $m e V^{-4}$ ) up to $T^{*}=18.339 K$; (b) Ground state fluctuation eigenvalue (units $m e V^{2}$ ) up to $T_{c}^{*}$.

The physically relevant feature is that the $\varepsilon_{n}$ depend, through $p$, on the system size $L$ (or on $\left.T^{*}\right)$. However not all the $\varepsilon_{n}$ are good fluctuation eigenvalues. In fact the first four values have to be discarded as their eigenfunctions do not fulfill the PBC required for the fluctuation components: $\eta_{n}\left(\varpi_{1}\right)=\eta_{n}\left(\varpi_{1} \mp 2 K(p)\right)$. Thus, three good eigenvalues in polynomial form are found: i) $\varepsilon_{0}=0$ is the zero mode eigenvalue which is consistently found also through the stability equation. ii) $\varepsilon_{1}$ lies in the continuum and vanishes at $p=0$ : this is the soft mode responsible for the sharp drop observed in $\operatorname{Det}^{R}[\hat{O}]$ as the sphaleron is approached. At $T_{c}^{*}, \varepsilon_{1}$ and $\varepsilon_{0}$ merge consistently with the double degeneracy of the corresponding eigenmodes above the crossover. iii) $\varepsilon_{-1}$ is the negative eigenvalue responsible for the decay of the metastable state. In Fig. 7, the ratio $\operatorname{Det}^{R}[\hat{O}] / \varepsilon_{-1}$ and $\varepsilon_{-1}$ are plotted separately to emphasize the role of the latter. The dependence on $T^{*}$ is obtained as usual by means of Eq. (13). While the overall shape of $\operatorname{Det}^{R}[\hat{O}]$ is not essentially modified by extracting $\varepsilon_{-1}$ (compare with Fig. (5), the interesting feature is represented by the softening of $\varepsilon_{-1}$ with respect to the value $\varepsilon_{-1}=-5 \omega^{2} / 4$ found at $T^{*}=0$. The substantial reduction (in absolute value) starts up at $T^{*} \sim 10 K$, that is in the same range at which the classical properties deviate from the predictions of the zero temperature theory. Finally, at the sphaleron, from Eq. (33) I get $\varepsilon_{-1}=-\omega^{2}$ consistently with the observation made at the end of the previous Section. This completes the analysis of the quantum fluctuation spectrum. 


\section{Decay Rate}

The decay rate in Eq. (1) for the finite temperature theory can be now computed as the

coefficients $A$ and $B$, identified with $\sqrt{|\operatorname{Det}[\hat{h}] / \operatorname{Det}[\hat{O}]|} / L$ and $A\left[x_{c l}\right]$ respectively, have been analysed in detail. The general expression for $\Gamma\left(T^{*}\right)$ is:

$$
\Gamma\left(T^{*}\right)=\hbar \sqrt{\frac{M}{2 \pi \hbar}} \bar{N}^{-1} \sqrt{\frac{\operatorname{Det}[\hat{h}]}{\operatorname{Det}^{R R}[\hat{O}]\left|\varepsilon_{-1}\right|}} \exp \left[-\frac{A\left[x_{c l}\right]}{\hbar}\right]
$$

with $\operatorname{Det}^{R R}[\hat{O}] \equiv \operatorname{Det}^{R}[\hat{O}] / \varepsilon_{-1}$. Essentially, the two bound state eigenvalues $\varepsilon_{0}$ and $\varepsilon_{-1}$ have been extracted from $\operatorname{Det}[\hat{O}]$ and treated separately.

It is now clear that, in order to calculate the decay rate, there would be no need to compute $\bar{N}^{-1}$ due to the zero mode [40] as it cancels out with the same term embedded in $D e t^{R}[\hat{O}]$ and discussed above. Thus, it is the piece of determinant in Fig. [6(a) which enters the computation of $\Gamma$.

Eq. (34) is plotted in Fig. 8 against temperature up to $T_{c}^{*}=18.469 \mathrm{~K}$ together with the constant decay rate of the zero temperature theory. There is a substantial increase in $\Gamma$ above $T^{*} \sim 10 K$ where the combined effects of quantum fluctuations and classical action softening become evident. Approaching the crossover (at $T_{P}^{*}=18.138 K$ ), $\Gamma$ reaches a peak value which is larger by a factor 5 with respect to the value of the zero $T$ theory although the linewidth remains much smaller than the oscillator energy, $\Gamma\left(T_{P}^{*}\right) / \hbar \omega \sim 10^{-5}$. Certainly, such ratio strongly depends on the particle mass through the exponential Gamow factor as $A\left[x_{c l}\right] \propto M$. Beyond $T_{P}^{*}$ and before $T_{c}^{*}$, the quantum decay rate smoothly merges with the classical Arrhenius factor as $A\left[x_{c l}\right] / \hbar \rightarrow V(a) / K_{B} T_{c}^{*}$. In principle this should occur exactly at $T_{c}^{*}$ once the nonuniform bounce leaves room to the constant solution $x_{c l}(\tau)=a$. But close to $T_{c}^{*}$ the decay rate drops to zero as $\Gamma\left(T^{*}\right) \propto\left(T_{c}^{*}-T^{*}\right)^{1 / 2}$ signalling that the quantum tunneling effects is ending. The power law dependence is driven by $\sqrt{N^{-2} / \operatorname{Det}^{R}[\hat{O}]} \propto p$. For this reason the Arrhenius behavior in the decay rate, which is a continuous function of $T^{*}$, takes over slightly before $T_{c}^{*}$ and precisely at $T_{A}^{*}=18.25 \mathrm{~K}$ corresponding to the symbol in Fig. 8.

The inset shows the plot of the quantum statistical decay rate $\Gamma_{\Sigma}[13]$ which I have computed, on the base of the WKB turning point formula [12], by summing over the particle 
energies in the cubic potential case. In the quantum regime, the calculation converges by taking about 1000 energy values (below the sphaleron $V(a)$ ) in the Boltzmann average. In the activated regime (with energies above the sphaleron), $\Gamma_{\Sigma}$ continuously grows with exponential behavior while, the matching between the quantum and activated $\Gamma_{\Sigma}$ plots occurs at around $T_{c}^{*}$, with states of energy $E \sim V(a)$ yielding a significant contribution to $\Gamma_{\Sigma}$ in the classical regime.

The deviation with respect to the $T=0$ result found in the quantum decay rate $\Gamma\left(T^{*}\right)$ (and culminating in the peak value) is interesting also in view of a comparison with classically activated systems in which spatio-temporal noise induces transitions between locally stable states of a nonlinear potential. The changes in radius and the stability conditions of metastable metallic nanowires are an example of current interest [17, 41, 42]. In these systems, the finite size shapes the activation behavior and a power-law divergence in the escape rate with critical exponent $1 / 2$ is predicted once a critical lenghtscale is approached.

In the decay rate of Eq. (34) the small parameter is $\hbar$ which, unlike the noise in classical systems, cannot be varied as a function of $T$ (or $L$ ). Accordingly the quantum tunneling decay rate is always small. Similar conclusions regarding the transition can be drawn by carrying out the study of $\Gamma$ for the metastable quartic potential

$$
V(x)=\frac{M \omega^{2}}{2} x^{2}-\frac{\delta}{4} x^{4}
$$

according to the same semiclassical method described so far. The parameters $M, \omega$ and $a$ have been taken as in the cubic case while $\delta$, in units $\mathrm{eV} \AA^{-4}$, is given by $\delta=M \omega^{2} / a^{2}$. There are in fact some fundamental differences which may be summarized as follows: 1) for the quartic potential, the bounce solution is linear in the Jacobi elliptic $d n$-amplitude and the crossover temperature $T_{c}^{*}$ is larger by a factor $\sqrt{2}$ with respect to the value given in Eq. (14). 2) The classical action is slightly larger than that computed in Fig. 4 and, in the $E \rightarrow 0$ limit, $A\left[x_{c l}\right] \rightarrow 4 M^{2} \omega^{3} /(3 \hbar \delta)$. 3) The harmonic oscillator over regularized fluctuations determinants ratio is smaller and, in the $T^{*} \rightarrow 0$ limit: $\operatorname{Det}[\hat{h}] / \operatorname{Det}^{R}[\hat{O}] \rightarrow-12 \omega^{2}$. 4) The Lamè equation, which has to be solved for the case $l=2$, has the negative eigenvalue scaling from $\varepsilon_{-1}=-3 \omega^{2}$ at $T^{*}=0$ to $\varepsilon_{-1}=-2 \omega^{2}$ at $T^{*}=T_{c}^{*}$. All these features work together to decrease the decay rate of the metastable quartic potential with respect to the cubic one. The quantitative results are reported on in Fig. 9 by plotting $\Gamma$ for the two potentials. 


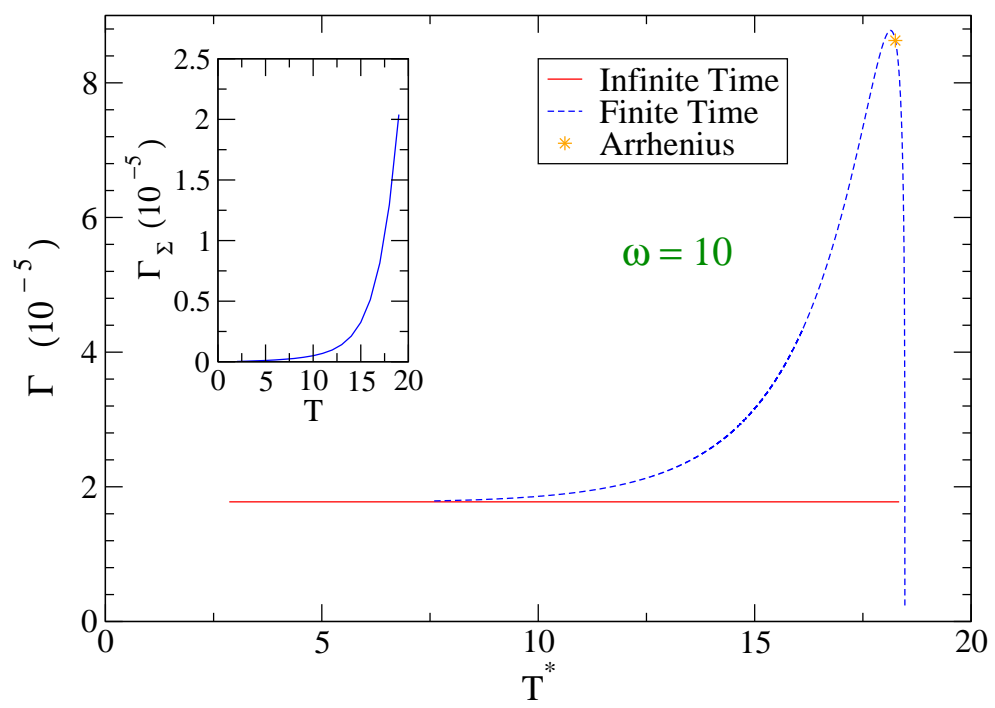

FIG. 8: (Color online) Decay rate (in $m e V$ ) for a particle in a metastable cubic potential versus temperature up to $T_{c}^{*}$. The result of the standard instantonic theory is shown for comparison. The symbol marks the point in which the quantum decay rate merges with the classical Arrhenius factor. The quantum statistical decay rate $\Gamma_{\Sigma}$ is plotted in the inset.

It appears that, at a given $T^{*}$ within the quantum regime, the life of a particle set in a metastable quartic potential is longer (by an order of magnitude) than in a cubic potential with the same structural parameters. Close to $T_{c}^{*}$, the decay rate of the quartic potential shows the same behavior found in the cubic case as a consequence of the softening of the polynomial fluctuation eigenmode with lowest lying positive eigenvalue.

\section{Conclusion}

The semiclassical path integral method has been applied to compute the decay rate of a metastable cubic potential at finite temperature. I have considered a non dissipative system where the inverse temperature, introduced as the imaginary time of the Euclidean path integral, represents a measure of the system size. Solving the Euler-Lagrange equation for the cubic potential I have determined the classical background, the bounce, in terms of Jacobi 


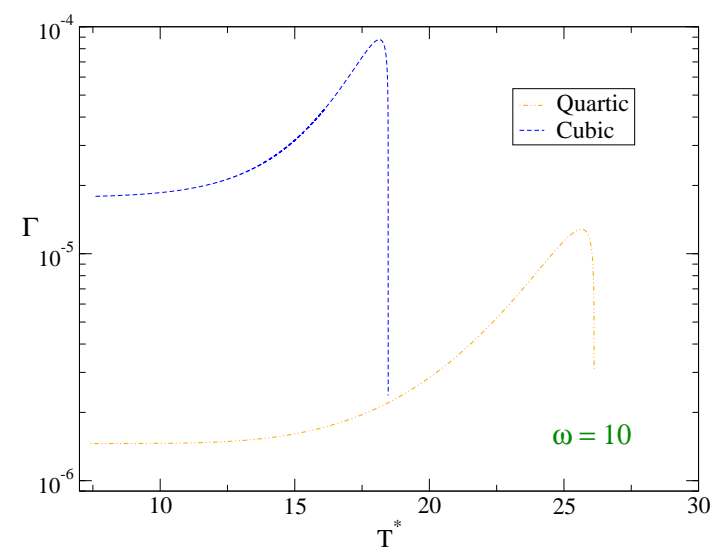

FIG. 9: (Color online) Decay rates of metastable cubic and quartic potentials versus temperature up to $T_{c}^{*}$.

elliptic functions whose energy dependent modulus keeps track of the classical mechanics. The periodicity property of the elliptic solution sets the fundamental relation between bounce oscillation period and classical energy. As the former varies monotonically with the latter, the transition from the quantum to the activated regime is in fact a temperature driven smooth crossover occuring at $T_{c}^{*} \propto \omega$. Then the quantum regime may cover a broader temperature range in systems with characteristic frequencies larger than the value taken here. In the latter cases however the quadratic approximation for the quantum fluctuations may become questionable.

In general, the character of this transition in tunneling systems may depend on the form of the potential. For the metastable potential the classical background represented by the bounce has zero topological charge, fulfills periodic boundary conditions and the amplitude of its motion progressively shrinks by increasing $T^{*}$. At $T_{c}^{*}$, the bounce degenerates into a point, the constant solution which determines the classical Arrhenius factor. Then the nonuniform bounce samples a $T^{*}$ dependent portion of the (reversed) potential valley and continuosly adapts its shape to join the turning points. That's why the crossover is smooth.

As in the semiclassical method classical and quantum degrees of freedom are intertwinned the quantum fluctuation spectrum can be evaluated, by the theory of the functional determinants, in terms of the classical bounce. More precisely, in terms of its time and modulus derivatives. I have obtained a compact expression for the fluctuations contribution to the path integral and computed the renormalization of the fluctuation spectrum due to temperature effects inside the quantum regime. The overall softening is remarkable: it shows up 
at about $T_{c}^{*} / 2$ whereas at lower temperatures the predictions of the zero $T$ (infinite time) theory are accurate. The polynomial eigenmodes of the spectrum have been analytically determined by solving the stability equation associated to the Sturm-Liouville differential operator, the Lamè equation. The focus on the ground state is justified by the fact that its eigenvalue is negative hence, it is responsible for the decay rate $\Gamma$ of the metastable potential. The negative eigenvalue softens considerably by approaching the sphaleron where, in turn, it determines the oscillation period of the classical bounce. Also the positive polynomial eigenvalue plays a key role as it even vanishes at the sphaleron and ultimately induces the rapid increase in the inverse determinants ratio. The explicitly derived equations governing the temperature dependence of the fluctuations may be adapted to specific situations by tuning the potential parameters. For the specific choice of parameters here presented, $M=10^{4} m_{e}$ and $\hbar \omega=10 \mathrm{meV}$, the computed $\Gamma$ starts to increase over the zero $T$ decay rate at about $T_{c}^{*} / 2$ and, close to $T_{c}^{*}$, the peak value is larger by a factor 5 , a sizeable effect. At the crossover the quantum decay process ends and the Arrhenius classical behavior sets in. Finally, a comparison between cubic and quartic metastable potentials having the same structural parameters shows that the latter is less metastable in the sense that the lifetime is found to be larger by a factor ten at any temperature in the quantum regime.

[1] J.S.Langer, Ann. Phys. 41, 108 (1967).

[2] M.B.Voloshin, I.Y.Kobzarev, L.B.Okun, Sov. J. Nucl. Phys. 20, 644 (1975).

[3] T.Banks, C.Bender, T.T.Wu, Phys. Rev. D 8, 3346 (1973).

[4] S.Coleman, Phys. Rev. D 15, 2929 (1977); C.G.Callan, S.Coleman, Phys. Rev. D 16, 1762 (1977).

[5] L.D.Landau, E.M.Lifshitz, Quantum Mechanics $3^{\text {rd }}$ edn. (Butterworth-Heinemann, Oxford, 1977).

[6] R.P.Feynman, Rev. Mod. Phys. 20, 367 (1948).

[7] L.S.Schulman Techniques and Applications of Path Integration (Wiley\&Sons, New York, 1981).

[8] I.M.Gelfand, A.M.Yaglom, J.Math.Phys. 1, 48 (1960).

[9] M.Zoli, Phys. Rev. B, 72, 214302 (2005). 
[10] R.Rajaraman, Solitons and Instantons (North Holland, Amsterdam, 1982).

[11] E.Brézin, G.Parisi, J.Zinn-Justin, Phys. Rev. D 16, 408 (1977).

[12] I.Affleck, Phys. Rev. Lett. 46, 388 (1981).

[13] U.Weiss, W.Haeffner, Phys. Rev. D 27, 2916 (1983).

[14] M.B.Voloshin, Phys. Lett. B 599, 129 (2004).

[15] H.Kleinert, Path Integrals in Quantum Mechanics, Statistics, Polymer Physycs and Financial Markets (World Scientific Publishing, Singapore, 2004).

[16] H.-B.Braun, Phys. Rev. Lett. 71, 3557 (1993).

[17] J.Bürki, C.A.Stafford, D.L.Stein, Phys. Rev. Lett. 95, 090601 (2005).

[18] W.G.Faris, G.Jona-Lasinio, J. Phys. A 15, 3025 (1982).

[19] The concept of temperature needs a clarification. In theories of phase transitions with symmetry breaking the effective potential is a function of the temperature. By cooling the system, a minimum (corresponding to a non-zero Higgs field $\varphi$ ) appears in the potential. Thus the metastable state placed at $\varphi=0$ decays via quantum tunneling to the $\varphi \neq 0$ energetically favourable state. Here I'm taking a specific potential which does not change its shape with $T$ : it has a metastable state at any $T$ within the considered quantum regime. Instead, it is the particle moving in such potential which feels the temperature effects. The bounce plus its quantum fluctuations depend on $T$ which is here an inverse time.

[20] Z.X.Wang, D.R.Guo, Special Functions (World Scientific, Singapore, 1989).

[21] P.Hänggi, P.Talkner, M.Borkovec, Rev. Mod. Phys. 62, 251 (1990).

[22] N.Antunes, F.C.Lombardo, D.Monteoliva, P.I.Villar, Phys. Rev. E 73, 066105 (2006).

[23] P.S.Riseborough, P.Hänggi, E.Freidkin, Phys. Rev. A 32, 489 (1985).

[24] D.L.Stein, Brazilian J. Phys. 35, 242 (2005).

[25] N.S.Manton, Phys. Rev. D 28, 2019 (1983).

[26] I.S.Gradshteyn, I.M.Ryzhik, Tables of Integrals, Series and Products (Academic Press, New York, 1965).

[27] V.I.Goldanskii, Sov.Phys.Dokl. 4, 74 (1959).

[28] A.I.Larkin, Y.N.Ovchinnikov, Sov.Phys.JETP 37, 382 (1983); ibid. 59, 420 (1984).

[29] H.Grabert, U.Weiss, Phys. Rev. Lett. 53, 1787 (1984).

[30] D.A.Gorokhov, G.Blatter, Phys. Rev. B 56, 3130 (1997).

[31] A.N.Kuznetsov, P.G.Tinyakov, Phys. Lett. B 406, 76 (1997). 
[32] J.-Q.Liang, H.J.W.Müller-Kirsten, D.K.Park, F.Zimmerschied, Phys. Rev. Lett. 81, 216 (1998).

[33] E.M.Chudnovski, Phys. Rev. A 46, 8011 (1992).

[34] This can be directly deduced from Eq. (12) as $K(p=0)=\pi / 2$.

[35] S.Y.Lee, H.Kim, D.K.Park, J.K.Kim, Phys. Rev. B 60, 10086 (1999).

[36] R.Forman, Invent.Math. 88, 447 (1987); ibid. Commun. Math. Phys. 147, 485 (1992).

[37] K.Kirsten, A.J.McKane, J.Phys A:Math. Gen. 37, 4649 (2004).

[38] R.S.Ward, J.Phys.A: Math.Gen. 20, 2679 (1987).

[39] The way to order eigenvalues and eigenstates in terms of $n$ is purely conventional. Either $n \in[-l, l]$ or $n \in[0,2 l+1]$ can be used. The former is assumed here consistently with having labelled the unstable eigenvalue by $n=-1$.

[40] A.J.McKane, M.B.Tarlie, J.Math.Phys. 28, 6931 (1995).

[41] A.I.Yanson, I.K.Yanson, J.M. van Ruitenbeck, Nature 400, 144 (1999).

[42] C.-H.Zhang, F.Kassubek, C.A.Stafford, Phys. Rev. B 68, 165414 (2003). 